\title{
CONTROL BY INTERCONNECTION FOR DISTRIBUTED PORT HAMILTONIAN SYSTEMS
}

\author{
Alessandro Macchelli * Arjan van der Schaft ${ }^{* *}$ \\ Claudio Melchiorri* \\ * CASY-DEIS, University of Bologna, \\ viale Risorgimento 2, 40136 Bologna, Italy \\ ** Dept. of Applied Mathematics, University of Twente, \\ 7500 AE Enschede, The Netherlands
}

\begin{abstract}
In this paper, new results on the control of distributed parameter systems in port Hamiltonian form are presented. The control by interconnection and energy shaping is applied to the stabilization of a distributed parameter system by means of a finite dimensional controller. The regulator acts on the system through the boundary or the distributed port. The key point is the generalization of Casimir function for systems resulting from the power conserving interconnection of an infinite and a finite dimensional part. Copyright (C) 2005 IFAC
\end{abstract}

Keywords: Port-Hamiltonian systems, Infinite-dimensional systems, Energy-shaping

\section{INTRODUCTION}

The port Hamiltonian description of finite dimensional systems (Maschke and van der Schaft, 1992) has been generalized to the distributed parameter case, (Macchelli et al., 2004a; van der Schaft and Maschke, 2002) by extending the notion of Dirac structure (Dalsmo and van der Schaft, 1999) on an infinite dimensional power space.

In this paper, the control by interconnection is generalized to the regulation of an infinite dimensional system by means of a finite dimensional boundary or distributed controller. The main result concerns the conditions for a real-valued function defined over the state space of the closed loop system to be a structural invariant (Casimir function). Once these conditions are deduced, by choosing a proper family of Casimir functions, the control by interconnection and energy shaping methodology can be applied as in the finite dimensional case. In this way, the open-loop energy function can be shaped by introducing a new minimum at the desired equilibrium configuration.

This paper is organized as follows. In Sect. 2, the control by interconnection and energy shaping for finite dimensional systems is briefly introduced. Then, the boundary control by interconnection for infinite dimensional systems is discussed in Sect. 3, while the distributed control is presented in Sect. 4. In both cases, conditions for the existence of Casimir functions in the closed loop system are deduced and the application in the energy shaping procedure is described. Finally, conclusions are presented in Sect. 5 .

\section{CONTROL BY INTERCONNECTION IN FINITE DIMENSIONS}

Denote by $\mathcal{X}$ an $n$-dimensional space of state (energy) variables and by $H: \mathcal{X} \rightarrow \mathbb{R}$ the energy (Hamiltonian) function, bounded from be- 
low. Moreover, denote by $\mathcal{U}$ an $m$-dimensional (linear) space of input variables and by its dual $\mathcal{Y} \equiv \mathcal{U}^{*}$ the space of output variables. Then,

$$
\left\{\begin{array}{l}
\dot{x}=[J(x)-R(x)] \frac{\partial H}{\partial x}+G(x) u \\
y=G^{\mathrm{T}}(x) \frac{\partial H}{\partial x}
\end{array}\right.
$$

with $J(x)=J^{\mathrm{T}}(x), R(x)=R^{\mathrm{T}}(x) \geq 0$ and $G(x)$ matrices of proper dimensions, is a port Hamiltonian system with dissipation, (Maschke and van der Schaft, 1992). $J$ and $R$ are called interconnection and damping matrices respectively.

Suppose that (1) has to be asymptotically stabilized around the configuration $x^{*} \in \mathcal{X}$ by means of the following dynamical controller in port Hamiltonian form:

$$
\left\{\begin{array}{l}
\dot{x}_{c}=\left[J_{c}\left(x_{c}\right)-R_{c}\left(x_{c}\right)\right] \frac{\partial H_{c}}{\partial x_{c}}+G_{c}\left(x_{c}\right) u_{c} \\
y_{c}=G_{c}^{\mathrm{T}}\left(x_{c}\right) \frac{\partial H_{c}}{\partial x_{c}}
\end{array}\right.
$$

Denote by $\mathcal{X}_{c}$ the controller state space, with $\operatorname{dim} \mathcal{X}_{c}=n_{c}$, and by $H_{c}: \mathcal{X}_{c} \rightarrow \mathbb{R}$ the Hamiltonian function, bounded from below. Moreover, suppose that $J_{c}\left(x_{c}\right)=-J_{c}^{\mathrm{T}}\left(x_{c}\right)$ and $R_{c}\left(x_{c}\right)=$ $R_{c}^{\mathrm{T}}\left(x_{c}\right) \geq 0$ and that $\operatorname{dim} \mathcal{U}_{c}=\operatorname{dim} \mathcal{Y}_{c}=m$.

If systems (1) and (2) are interconnected in power conserving way, that is if

$$
\left\{\begin{array}{l}
u=-y_{c} \\
y=u_{c}
\end{array}\right.
$$

the resulting closed loop system is given by an autonomous port Hamiltonian system, with state space $\mathcal{X} \times \mathcal{X}_{c}$ and Hamiltonian $H+H_{c}$.

Given a generic port Hamiltonian system, it is possible to give the following fundamental definition of structural invariant or, equivalently, of Casimir function, (Dalsmo and van der Schaft, 1999; Marsden and Ratiu, 1994; van der Schaft, 2000).

Definition 1. Consider the port Hamiltonian system (1) with state space $\mathcal{X}$ and Hamiltonian function $H: \mathcal{X} \rightarrow \mathbb{R}$. A function $\mathcal{C}: \mathcal{X} \rightarrow \mathbb{R}$ is a Casimir function for (1) if and only if $\dot{\mathcal{C}}=0$ along system trajectories for every possible choice of Hamiltonian $H$.

The existence of Casimir functions for the closed loop system plays an important role in the control by interconnection and energy shaping methodology. If $x^{*} \in \mathcal{X}$ is the desired equilibrium configuration for (1), asymptotic stability in $x^{*}$ can be achieved by properly choosing the Hamiltonian function of (2) in order to shape the closed-loop energy $H+H_{c}$ so that a (possibly) global minimum in the desired equilibrium configuration can be introduced. It is important to note that there is no relation between the state of the controller and the state of the system to be controlled. Then, it is not clear how the controller energy, which is freely assignable, has to be chosen in order to solve the regulation problem.

A possible solution can be to constrain the state of the closed-loop system on a certain subspace of $\mathcal{X} \times \mathcal{X}_{c}$, for example given by:

$$
\Omega_{c}:=\left\{\left(x, x_{c}\right) \in \mathcal{X} \times \mathcal{X}_{c} \mid x_{c}=S(x)+c\right\}
$$

where $c \in \mathbb{R}^{n_{c}}$ and $S: \mathcal{X} \rightarrow \mathcal{X}_{c}$ is a function to be computed. In other words, we are looking for a set of Casimir functions $\mathcal{C}_{i}: \mathcal{X} \times \mathcal{X}_{c} \rightarrow \mathbb{R}$, $i=1, \ldots, n_{c}$ for the closed loop system such that

$$
\mathcal{C}_{i}\left(x, x_{c}\right):=S_{i}(x)-x_{c, i}
$$

where $\left[S_{1}(x), \ldots, S_{n_{c}}(x)\right]^{\mathrm{T}}=S(x)$. Due to the nature of a Casimir function, it is possible to introduce an intrinsic non-linear state feedback law that will be used in order to choose the energy function of the controller so that the closed loop Hamiltonian can be properly shaped. Note that, under these hypothesis, this energy function depends on the state variables of system (1). This control methodology is called invariant function method, (Dalsmo and van der Schaft, 1999; Marsden and Ratiu, 1994). The following proposition can be proved, (Ortega et al., 1999; van der Schaft, 2000).

Proposition 2. The functions $\mathcal{C}_{i}, i=1, \ldots, n_{c}$, defined in (4) are Casimir functions for the closed loop system if and only if the following conditions are satisfied:

$$
\begin{aligned}
\frac{\partial^{\mathrm{T}} S}{\partial x} J(x) \frac{\partial S}{\partial x} & =J_{c}\left(x_{c}\right) \\
R(x) \frac{\partial S}{\partial x} & =0 \\
R_{c}\left(x_{c}\right) & =0 \\
\frac{\partial^{\mathrm{T}} S}{\partial x} J(x) & =G_{c}\left(x_{c}\right) G^{\mathrm{T}}(x)
\end{aligned}
$$

Suppose that (5) are satisfied. Then, from (4), the state variables of the controller are robustly related to the state variable of the system to be stabilized since

$$
x_{c, i}=S_{i}(x)+c_{i}, \quad i=1, \ldots, n_{c}
$$

with $c_{i} \in \mathbb{R}$ depending only on the initial conditions. Moreover, the closed loop dynamics becomes:

$$
\dot{x}=[J(x)-R(x)]\left(\frac{\partial H}{\partial x}+\frac{\partial S}{\partial x} \frac{\partial H_{c}}{\partial x_{c}}\right)
$$

From (6), we have that $H_{c}\left(x_{c}\right) \equiv H_{c}(S(x)+c)$ : finally, the controller energy function depends on $x$ through the non-linear feedback action $S(\cdot)$. If

$$
H_{d}(x):=H(x)+H_{c}(S(x)+c)
$$


then (7) can be written as

$$
\dot{x}=[J(x)-R(x)] \frac{\partial H_{d}}{\partial x}
$$

By properly choosing the controller energy function $H_{c}$, it is possible to shape the closed loop energy function $H_{d}$ defined in (8) so that a new minimum in $x^{*}$ is introduced. Then, the desired configuration can be reached with the dynamics given by (9), (Ortega et al., 1999; van der Schaft, 2000).

\section{BOUNDARY CONTROL BY INTERCONNECTION OF MDPH SYSTEMS}

In this section, the control by interconnection and energy shaping, discussed in Sect. 2 for the finite dimensional case, is generalized to distributed parameter systems in port Hamiltonian form. In particular, it is shown how it is possible to shape the open loop energy function by interconnecting a finite dimensional controller to its boundary. The generalization to the case in which the controller is interconnected to the distributed port is presented in Sect. 4.

\subsection{Existence of Casimir functions}

Consider the following multi-variable distributed port Hamiltonian system with spatial domain $\mathcal{Z} \subset \mathbb{R}^{d}$ (closed and compact), (Macchelli et al., 2004a):

$$
\left\{\begin{aligned}
\frac{\partial x}{\partial t} & =(J-R) \delta_{x} \mathcal{H} \\
w & =B_{\mathcal{Z}}\left(\delta_{x} \mathcal{H}\right)
\end{aligned}\right.
$$

where $x \in \mathcal{X}$ is the configuration variable, $w \in \mathcal{W}$ are the boundary terms defined by the boundary operator $B_{\mathcal{Z}}, \mathcal{H}: \mathcal{X} \rightarrow \mathbb{R}$ is the Hamiltonian function, $J$ is a skew adjoint differential operator and $R$ is a non-negative self-adjoint differential operator taking into account the dissipative effects. Both $\mathcal{X}$ either $\mathcal{W}$ are spaces of vector value smooth functions of proper dimension.

It is possible to prove that the following energy balance relation holds, (Macchelli et al., 2004a):

$$
\frac{\mathrm{d} \mathcal{H}}{\mathrm{d} t} \leq \frac{1}{2} \int_{\partial \mathcal{Z}} B(w, w) \cdot \mathrm{dA}
$$

where the integral over $\partial \mathcal{Z}$ represent the power exchanged with the environment through the boundary and the $B$ is a constant operator depending on the differential operators $J$ and $R$. See (Macchelli et al., 2004a) for more details.

Suppose that (10) has to be stabilized in the configuration $x^{*} \in \mathcal{X}$ by means of the finite dimensional controller (2) that has to be interconnected to the system (10) at the boundary in power conserving way. Then, relation (3) has to be generalized in order to deal with a situation in which the power port of the system to be stabilized is not a finite dimensional vector space. A possible solution can be the following. Denote by $\Psi_{u}(z)$ and $\Psi_{y}(z)$ a couple of matrices depending eventually on $z \in \partial \mathcal{Z}$ and suppose that it is possible to write the boundary terms in (10) as $w=\Psi_{u} u+\Psi_{y} y$ with $u \in \mathcal{U}$ and $y \in \mathcal{Y}$, where $\mathcal{U}$ and $\mathcal{Y}$ are finite dimensional linear spaces in duality. Consequently, if (3) holds, it is possible to write the boundary terms in (10) as follows:

$$
w=\Psi_{y} u_{c}-\Psi_{u} y_{c}
$$

The interconnection law expressed in (12) is power conserving if and only if

$$
y_{c}^{\mathrm{T}} u_{c}+\frac{1}{2} \int_{\partial \mathcal{Z}} B(w, w) \cdot \mathrm{dA}=0
$$

or, equivalently, if

$$
\begin{aligned}
& \int_{\partial \mathcal{Z}} B\left(\Psi_{u, i}, \Psi_{u, j}\right) \cdot \mathrm{dA}=0 \\
& \int_{\partial \mathcal{Z}} B\left(\Psi_{y, i}, \Psi_{y, j}\right) \cdot \mathrm{dA}=0 \\
& \int_{\partial \mathcal{Z}} B\left(\Psi_{u, i}, \Psi_{y, j}\right) \cdot \mathrm{dA}=\delta_{i j}
\end{aligned}
$$

for every $i, j=1, \ldots, m$ and where $\delta$ is the Kronecker symbol. Note that, given $w \in \mathcal{W}$

$$
\begin{aligned}
u_{c, i} & =\int_{\partial \mathcal{Z}} B\left(\Psi_{u, i}, w\right) \cdot \mathrm{dA} \\
y_{c, i} & =-\int_{\partial \mathcal{Z}} B\left(\Psi_{y, i}, w\right) \cdot \mathrm{dA}
\end{aligned}
$$

or equivalently that

$$
u_{c}=\mathcal{B}^{u}(w) \quad y_{c}=-\mathcal{B}^{y}(w)
$$

where $\mathcal{B}^{u}: \mathcal{W} \rightarrow \mathcal{U}_{c}$ and $\mathcal{B}^{y}: \mathcal{W} \rightarrow \mathcal{Y}_{c}$ are two linear operator whose definition is based on (14).

Consider a function $\mathcal{C}: \mathcal{X} \times \mathcal{X}_{c} \rightarrow \mathbb{R}$ defined over the state space of the closed loop system resulting from the power conserving interconnection (12) of (10) and (2). From Def. 1, we can say that $\mathcal{C}$ is a Casimir function if and only if

$$
\begin{aligned}
\frac{\mathrm{d} \mathcal{C}}{\mathrm{d} t}= & \frac{\partial^{\mathrm{T}} \mathcal{C}}{\partial x_{c}}\left(J_{c}-R_{c}\right) \frac{\partial H_{c}}{\partial x_{c}}+\frac{\partial^{\mathrm{T}} \mathcal{C}}{\partial x_{c}} G_{c} \mathcal{B}^{u}(w) \\
& +\int_{\mathcal{Z}}\left(\delta_{x} \mathcal{C}\right)^{\mathrm{T}}(J-R) \delta_{x} \mathcal{H} \mathrm{dV}=0
\end{aligned}
$$

along system trajectories and for every Hamiltonian functions $\mathcal{H}$ and $H_{c}$, where $u_{c}$ is expressed as a function of the boundary terms as in (14). Since $J$ and $R$ are a skew adjoint and a self adjoint differential operator respectively, we have that (see (Macchelli et al., 2004a; Renardy and Rogers, 2004)):

$$
\begin{gathered}
\left(\delta_{x} \mathcal{C}\right)^{\mathrm{T}}(J-R) \delta_{x} \mathcal{H}=-\left(\delta_{x} \mathcal{H}\right)^{\mathrm{T}}(J+R) \delta_{x} \mathcal{C} \\
+\operatorname{div} B\left(B_{\mathcal{Z}}\left(\delta_{x} \mathcal{C}\right), w\right)
\end{gathered}
$$


and then

$$
\begin{gathered}
\frac{\mathrm{d} \mathcal{C}}{\mathrm{d} t}=-\frac{\partial^{\mathrm{T}} H_{c}}{\partial x_{c}}\left[\left(J_{c}+R_{c}\right) \frac{\partial \mathcal{C}}{\partial x_{c}}+G_{c} \mathcal{B}^{u}\left(B_{\mathcal{Z}}\left(\delta_{x} \mathcal{C}\right)\right)\right] \\
-\int_{\mathcal{Z}}\left(\delta_{x} \mathcal{H}\right)^{\mathrm{T}}(J+R) \delta_{x} \mathcal{C} \mathrm{dV}+\left[\mathcal{B}^{u}\left(B_{\mathcal{Z}}\left(\delta_{x} \mathcal{H}\right)\right)\right]^{\mathrm{T}} \times \\
\times\left(\mathcal{B}^{y}\left(B_{\mathcal{Z}}\left(\delta_{x} \mathcal{C}\right)\right)+G_{c}^{\mathrm{T}} \frac{\partial \mathcal{C}}{\partial x_{c}}\right)
\end{gathered}
$$

that has to be 0 along system trajectories and for every Hamiltonian function of the closed loop system. See (Macchelli et al., 2004b) for more details. This is true if and only if

$$
\begin{aligned}
\left(J_{c}+R_{c}\right) \frac{\partial \mathcal{C}}{\partial x_{c}}+G_{c} \mathcal{B}^{u}\left(B_{\mathcal{Z}}\left(\delta_{x} \mathcal{C}\right)\right) & =0 \\
(J+R) \delta_{x} \mathcal{C} & =0 \\
\mathcal{B}^{y}\left(B_{\mathcal{Z}}\left(\delta_{x} \mathcal{C}\right)\right)+G_{c}^{\mathrm{T}} \frac{\partial \mathcal{C}}{\partial x_{c}} & =0
\end{aligned}
$$

Proposition 3. Consider the closed loop system resulting from the power conserving interconnection (12) of the infinite dimensional system (10) with the finite dimensional controller (2). Denote by $\mathcal{X}$ and $\mathcal{X}_{c}$ the state space of the distributed parameter system and of the controller respectively. Then, a real value function $\mathcal{C}: \mathcal{X} \times \mathcal{X}_{c} \rightarrow \mathbb{R}$ is a Casimir function for the closed loop system with respect to the interconnection law (12) if and only the set of conditions (17) are satisfied.

Note 1. The necessary and sufficient conditions (17) on the existence of structural invariants in the closed loop system are the generalization of analogous conditions (see (Ortega et al., 1999)) in the finite dimensional case. In the hybrid case, the structural invariants have to satisfy the PDEs (17a) and (17c) in the controller/plant variables and the PDE (17b) in the spatial variable of the distributed parameter system. Note that the boundary conditions for (17b) have to be chosen in such a way that (17a) and (17c) are satisfied.

\subsection{Energy shaping via structural invariants}

The existence of a particular class of Casimir functions in the controlled system is of great interest in the energy shaping procedure. Also in the distributed parameter case, a possible solution is to choose the structure of the controller (2) in order to introduce a set of $\bar{n} \leq n_{c}$ structural invariants in the form (4), where now

$$
\mathcal{S}_{i}(x)=\int_{\mathcal{Z}} S_{i}(z, x) \mathrm{dV}
$$

with $i=1, \ldots, \bar{n}$. These functions are Casimir function for the closed loop system if and only if the set of conditions (17) are satisfied. In particular, denote by $\bar{J}_{c}, \bar{R}_{c}$ and $\bar{G}_{c}$ the sub-matrices of the interconnection, damping and input matrices of (2) corresponding to the first $\bar{n}$ state variables.
Then, from (17), we obtain the following set of conditions:

$$
\begin{aligned}
G_{c}\left[\mathcal{B}^{u}\left(\delta_{x} \mathcal{S}_{1}\right) \cdots \mathcal{B}^{u}\left(\delta_{x} \mathcal{S}_{\bar{n}}\right)\right] & =\bar{J}_{c}+\bar{R}_{c} \\
(J+R) \delta_{x} \mathcal{S}_{i} & =0 \\
{\left[\mathcal{B}^{y}\left(\delta_{x} \mathcal{S}_{1}\right) \cdots \mathcal{B}^{y}\left(\delta_{x} \mathcal{S}_{\bar{n}}\right)\right] } & =\bar{G}_{c}^{\mathrm{T}}
\end{aligned}
$$

with $i=1, \ldots, \bar{n}$ and where, in order to keep a lighter notation, $\mathcal{B}^{u}(\cdot)$ and $\mathcal{B}^{y}(\cdot)$ stand respectively for $\mathcal{B}^{u}\left(B_{\mathcal{Z}}(\cdot)\right)$ and $\mathcal{B}^{y}\left(B_{\mathcal{Z}}(\cdot)\right)$. Note that, from (18a) and (18c) only $\left(\bar{J}_{c}+\bar{R}_{c}\right)$ is determined by the set of functionals $\mathcal{S}_{i}$, while $(18 \mathrm{c})$ gives the expression of the input sub-matrix $\bar{G}_{c}$. Clearly, $\bar{J}_{c}$, $\bar{R}_{c}$ and $\bar{G}_{c}$ depend on $\mathcal{S}_{i}$, which have to be solution of the PDE (18b) whose boundary conditions have to be chosen in such a way that (18a) and (18c) are satisfied. If the set of conditions (18) can be satisfied, then the closed loop Hamiltonian function becomes $\mathcal{H}_{c l}\left(x, x_{c}\right)=\mathcal{H}(x)+H_{c}\left(x_{c, 1}, \ldots, x_{c, n_{c}}\right)=$ $\mathcal{H}(x)+H_{c}\left(\mathcal{S}_{1}(x), \ldots, \mathcal{S}_{\bar{n}}(x), \ldots, x_{c, n_{c}}\right)$, thus depending explicitly on the configuration variable of the distributed parameter system.

If $\bar{n}=n_{c}$, the closed loop Hamiltonian becomes

$$
\mathcal{H}_{c l}\left(x, x_{c}\right)=\mathcal{H}(x)+H_{c}\left(\mathcal{S}_{1}(x), \ldots, \mathcal{S}_{n_{c}}(x)\right)
$$

i.e. only a function of the configuration variable of the distributed parameter system. By properly choosing the controller energy function, it is possible to introduce a minimum at the desired equilibrium configuration that can be reached is some dissipative effect is present in the system. In particular, if in (10) $R=0$, that is no dissipative/diffusion phenomena are present in the infinite dimensional plant, it is convenient to chose the controller structure in order to have $\bar{n}<n_{c}$ Casimir function in the form (4) and then to introduce energy dissipation by acting on the remaining energy variables.

\section{DISTRIBUTED CONTROL BY INTERCONNECTION AND ENERGY SHAPING}

\subsection{Existence of Casimir functions}

Consider the mdpH system (10) and add a distributed power port. Denote by $u \in \mathcal{U} \subset \mathbb{R}^{m}$ an input signal acting on the system by modulating a certain time invariant profile $G(z), z \in \mathcal{Z}$, and by $y \in \mathcal{Y} \equiv \mathcal{U}^{*}$ the corresponding dual output. The resulting system is given by

$$
\left\{\begin{aligned}
\frac{\partial x}{\partial t} & =(J-R) \delta_{x} \mathcal{H}+G(z) u \\
y & =\int_{\mathcal{Z}} G^{\mathrm{T}}(z) \delta_{x} \mathcal{H} \mathrm{dV} \\
w & =B_{\mathcal{Z}}\left(\delta_{x} \mathcal{H}\right)
\end{aligned}\right.
$$

and the following energy balance relation holds:

$$
\frac{\mathrm{d} \mathcal{H}}{\mathrm{d} t} \leq \frac{1}{2} \int_{\partial \mathcal{Z}} B(w, w) \cdot \mathrm{dA}+y^{\mathrm{T}} u
$$


in which the integral over $\partial \mathcal{Z}$ provides the power flowing through the boundary while $y^{\mathrm{T}} u$ the power through the distributed port.

In the remaining part of this section, it is supposed that the power flow through the boundary is equal to zero. In order to properly chose the right set of boundary terms to be set to zero, the same methodology of Sect. 3.1 is followed. In particular, denote by $\mathcal{U}_{e}$ and $\mathcal{Y}_{e}$ a couple of finite dimensional linear spaces of proper dimension and by $\Psi_{u}$ and $\Psi_{y}$ a couple of linear operators, eventually dependent on $z \in \partial \mathcal{Z}$. Then, suppose that the boundary conditions $w \in \mathcal{W}$ can be written as

$$
w=\Psi_{u} u_{e}+\Psi_{y} y_{e}
$$

with the operators $\Psi_{u}$ and $\Psi_{y}$ satisfying conditions (13). In order to have zero power flow through the boundary, just set $u_{e}=0$, that is, from (15)

$$
\mathcal{B}^{y}(w)=0
$$

Clearly, $y_{e}=\mathcal{B}^{u}(w)$.

Denote by $x^{*} \in \mathcal{X}$ a desired equilibrium configuration for (19) and consider the finite dimensional stabilizing controller in port Hamiltonian form (2) that has to be interconnected in power conserving way, i.e. via (3), to (19). Consider a function $\mathcal{C}: \mathcal{X} \times \mathcal{X}_{c} \rightarrow \mathbb{R}$ defined over the state space of the closed loop system and such that

$$
B_{\mathcal{Z}}\left(\delta_{x} \mathcal{C}\right) \in \operatorname{Im}\left[\Psi_{u}: \Psi_{y}\right]
$$

that is the value at the boundary conditions of $\delta_{x} \mathcal{C}$ can be parameterized as $\delta_{x} \mathcal{H}$ in (12). As discussed in Sect. 2 and 3, the first step in the control by interconnection methodology is to find necessary and sufficient conditions for the existence of Casimir functions for the closed loop system. We have that

$$
\begin{aligned}
\frac{\mathrm{d} \mathcal{C}}{\mathrm{d} t}= & \frac{\partial^{\mathrm{T}} \mathcal{C}}{\partial x_{c}}\left(J_{c}-R_{c}\right) \frac{\partial H_{c}}{\partial x_{c}}+\frac{\partial^{\mathrm{T}} \mathcal{C}}{\partial x_{c}} G_{c} \int_{\mathcal{Z}} G^{\mathrm{T}} \delta_{x} \mathcal{H} \mathrm{dV} \\
& +\int_{\mathcal{Z}}\left(\delta_{x} \mathcal{C}\right)^{\mathrm{T}}\left[(J-R) \delta_{x} \mathcal{H}-G G_{c}^{\mathrm{T}} \frac{\partial H_{c}}{\partial x_{c}}\right] \mathrm{dV}
\end{aligned}
$$

that has to be 0 along system trajectories and for every Hamiltonian $\mathcal{H}$ and $H_{c}$. From (16), we have that

$$
\begin{aligned}
\frac{\mathrm{d} \mathcal{C}}{\mathrm{d} t} & =\int_{\mathcal{Z}}\left(\delta_{x} \mathcal{H}\right)^{\mathrm{T}}\left[G G_{c}^{\mathrm{T}} \frac{\partial \mathcal{C}}{\partial x_{c}}-(J+R) \delta_{x} \mathcal{C}\right] \mathrm{dV} \\
& -\int_{\mathcal{Z}} \frac{\partial^{\mathrm{T}} H_{c}}{\partial x_{c}} \times \\
& \times\left[\frac{1}{\operatorname{vol}(\mathcal{Z})}\left(J_{c}+R_{c}\right) \frac{\partial \mathcal{C}}{\partial x_{c}}+G_{c} G^{\mathrm{T}} \delta_{x} \mathcal{C}\right] \mathrm{dV} \\
& +\int_{\partial \mathcal{Z}} B\left(B_{\mathcal{Z}}\left(\delta_{x} \mathcal{C}\right), B_{\mathcal{Z}}\left(\delta_{x} \mathcal{H}\right)\right) \cdot \mathrm{dA}
\end{aligned}
$$

where $\operatorname{vol}(\mathcal{Z})=\int_{\mathcal{Z}} 1 \mathrm{dV}$ is the measure of the spatial domain $\mathcal{Z}$. From the hypothesis of zero power flow through the boundary and conditions (20) and (21), the integral over $\partial \mathcal{Z}$ is equal to

$$
\mathcal{B}^{u \mathrm{~T}}\left(B_{\mathcal{Z}}\left(\delta_{x} \mathcal{H}\right)\right) \mathcal{B}^{y}\left(B_{\mathcal{Z}}\left(\delta_{x} \mathcal{C}\right)\right)
$$

and consequently, $\dot{\mathcal{C}}=0$ along system trajectories and for every Hamiltonian if

$$
\begin{aligned}
\frac{1}{\operatorname{vol}(\mathcal{Z})}\left(J_{c}+R_{c}\right) \frac{\partial \mathcal{C}}{\partial x_{c}}+G_{c} G^{\mathrm{T}} \delta_{x} \mathcal{C} & =0 \\
G G_{c}^{\mathrm{T}} \frac{\partial \mathcal{C}}{\partial x_{c}}-(J+R) \delta_{x} \mathcal{C} & =0 \\
\mathcal{B}^{y}\left(B_{\mathcal{Z}}\left(\delta_{x} \mathcal{C}\right)\right) & =0
\end{aligned}
$$

Note that (22c) for $\delta_{x} \mathcal{C}$ is the same of (20) for $\delta_{x} \mathcal{H}$, which is sufficient for having zero power flow through the boundary. In particular, a sort of orthogonality conditions between output boundary terms and variational derivatives of the Casimir function is required.

Proposition 4. Consider the closed loop system resulting from the power conserving interconnection (3) of the infinite dimensional system (19), whose boundary conditions are given by (20), with the finite dimensional controller (2). Denote by $\mathcal{X}$ and $\mathcal{X}_{c}$ the state space of the distributed parameter system and of the controller respectively. Then, a real value function $\mathcal{C}: \mathcal{X} \times \mathcal{X}_{c} \rightarrow \mathbb{R}$ is a Casimir function for the closed loop system with respect to the interconnection law (3) if the set of conditions (22) are satisfied.

\subsection{Energy shaping via structural invariants}

Denote by $x^{*} \in \mathcal{X}$ a desired equilibrium configuration of (19). As already discussed in Sect. 2 and in Sect. 3.2, in order to shape the open loop energy function $\mathcal{H}$ of (19) such that a minimum in $x^{*} \in \mathcal{X}$ is introduced, the first step is to chose the controller (2) in such a way that a set of $\bar{n} \leq n_{c}$ structural invariants in the form (4) are present in the closed loop system.

Consequently, from Prop. 4, it is necessary and sufficient that the set of conditions (22) are satisfied. In particular, denote by $\bar{J}_{c}, \bar{R}_{c}$ and $\bar{G}_{c}$ the sub-matrices of the interconnection, damping and input matrices of (2) corresponding to the first $\bar{n}$ state variables and define $\mathcal{S}: \mathcal{X} \rightarrow \mathbb{R}^{\bar{n}}$ as $\mathcal{S}=\left[\begin{array}{llll}\mathcal{S}_{1} & \cdots & \mathcal{S}_{\bar{n}}\end{array}\right]^{\mathrm{T}}$. From (22), we have that

$$
\begin{aligned}
\frac{1}{\operatorname{vol}(\mathcal{Z})}\left(\bar{J}_{c}+\bar{R}_{c}\right)-\bar{G}_{c} G^{\mathrm{T}} \delta_{x} \mathcal{S} & =0 \\
G \bar{G}_{c}^{\mathrm{T}}+(J+R) \delta_{x} \mathcal{S} & =0 \\
\mathcal{B}^{y}\left(B_{\mathcal{Z}}\left(\delta_{x} \mathcal{S}_{i}\right)\right) & =0
\end{aligned}
$$

$i=1, \ldots, \bar{n}$, and then, from (21) and since (23c) imposes 0 power flow through the boundary $\partial \mathcal{Z}$ for the dynamics specified by each functional $\mathcal{S}_{i}$, we have that

$\bar{J}_{c}-\bar{R}_{c}=\int_{\mathcal{Z}}\left(\delta_{x} \mathcal{S}\right)^{\mathrm{T}} J \delta_{x} \mathcal{S} \mathrm{dV}+\int_{\mathcal{Z}}\left(\delta_{x} \mathcal{S}\right)^{\mathrm{T}} R \delta_{x} \mathcal{S} \mathrm{dV}$

This relation can be satisfied if and only if 


$$
\begin{aligned}
\int_{\mathcal{Z}}\left(\delta_{x} \mathcal{S}\right)^{\mathrm{T}} J \delta_{x} \mathcal{S} \mathrm{dV} & =\bar{J}_{c} \\
R \delta_{x} \mathcal{S} & =0 \\
\bar{R}_{c} & =0
\end{aligned}
$$

since $\bar{J}_{c}=-\bar{J}_{c}^{\mathrm{T}}$ and $\bar{R}_{c}=\bar{R}_{c}^{\mathrm{T}} \geq 0$. The couple of relations (24a) and (24c) gives the expressions of the interconnection and damping matrices of the controller (2), while the set of PDEs (24b) provides the expression of the (nonlinear) feedback actions $\mathcal{S}_{i}, i=1, \ldots, \bar{n}$. The boundary conditions are imposed by (23c). Moreover, from (23b), (24a) and $(24 \mathrm{~b})$, we also deduce a relation that the input matrix $\bar{G}_{c}$ of the controller (2) has to satisfy:

$$
\bar{G}_{c} \int_{\mathcal{Z}} G^{\mathrm{T}} \delta_{x} \mathcal{S} \mathrm{dV}=\bar{J}_{c}
$$

If all the controller state variables are related to the configuration variables of the distributed parameter system via a set of (nonlinear) functionals, that is in the case $\bar{n}=n_{c}$, the closed loop dynamics can be written as

$$
\begin{aligned}
\frac{\partial x}{\partial t} & =(J-R) \delta_{x} \mathcal{H}-G G_{c}^{\mathrm{T}} \frac{\partial H_{c}}{\partial x_{c}} \\
& =(J-R) \delta_{x} \mathcal{H}_{c l}
\end{aligned}
$$

since (24b) holds and with

$$
\mathcal{H}_{c l}(x)=\mathcal{H}(x)+H_{c}\left(\mathcal{S}_{1}(x), \ldots, \mathcal{S}_{n_{c}}(x)\right)
$$

As regard the boundary conditions, we have that

$$
\begin{aligned}
w & =B_{\mathcal{Z}}\left(\delta_{x} \mathcal{H}_{c l}\right) \\
& =B_{\mathcal{Z}}\left(\delta_{x} \mathcal{H}\right)+\sum_{i=1}^{n} B_{\mathcal{Z}}\left(\delta_{x} \mathcal{S}_{i}\right) \frac{\partial H_{c}}{\partial x_{c, i}}
\end{aligned}
$$

Since, from (23c), we have that

$$
B_{\mathcal{Z}}\left(\delta_{x} \mathcal{S}_{i}\right) \in \operatorname{Ker} \mathcal{B}^{y} \quad i=1, \ldots, \bar{n}
$$

from condition (20), we deduce that

$$
\mathcal{B}^{y}\left(B_{\mathcal{Z}}\left(\delta_{x} \mathcal{H}_{c l}\right)\right)=0
$$

which is the constraint that the boundary terms of the closed loop dynamics has to satisfy. Finally, the following energy balance relation holds:

$$
\frac{\mathrm{d} \mathcal{H}_{c l}}{\mathrm{~d} t}=-\int_{\mathcal{Z}}\left(\delta_{x} \mathcal{H}_{c l}\right)^{\mathrm{T}} R \delta_{x} \mathcal{H}_{c l} \mathrm{dV} \leq 0
$$

\section{CONCLUSIONS AND FUTURE WORK}

In this paper, some new results on the control by interconnection and energy shaping of distributed parameter systems in port Hamiltonian form have been presented. The controller is a finite dimensional system that can act on the system through the boundary or a distributed port. The key point is the generalization of the necessary and sufficient conditions for having Casimir function in the hybrid case, i.e. when the closed loop system results from the power conserving interconnection of an infinite and a finite dimensional part.

\section{ACKNOWLEDGEMENTS}

This work has been done in the context of the European sponsored project GeoPlex, reference code IST-2001-34166. Further information is available at http://www.geoplex.cc.

\section{REFERENCES}

Dalsmo, M. and A. J. van der Schaft (1999). On representation and integrability of mathematical structures in energy-conserving physical systems. SIAM J. Control and Optimization (37), 54-91.

Macchelli, A., A. J. van der Schaft and C. Melchiorri (2004a). Port Hamiltonian formulation of infinite dimensional systems. I. Modeling. In: Proc. 50th IEEE Conference on Decisions and Control (CDCO4).

Macchelli, A., A. J. van der Schaft and C. Melchiorri (2004b). Port Hamiltonian formulation of infinite dimensional systems. II. Boundary control by interconnection. In: Proc. 50th IEEE Conference on Decisions and Control (CDCO4).

Marsden, J. E. and T. S. Ratiu (1994). Introduction to mechanics and symmetry. Springer, New York.

Maschke, B. M. and A. J. van der Schaft (1992). Port controlled Hamiltonian systems: modeling origins and system theoretic properties. In: Proceedings of the third Conference on nonlinear control systems (NOLCOS).

Ortega, R., A. J. van der Schaft, B. M. Maschke and G. Escobar (1999). Energy-shaping of port-controlled Hamiltonian systems by interconnection. In: Proc. IEEE Conf. Dec. and Control.

Renardy, M. and R. C. Rogers (2004). An Introduction to Partial Differential Equations. number 13 In: Texts in Applied Mathematics. 2nd ed.. Springer Verlag.

van der Schaft, A. J. (2000). $L_{2}$-Gain and Passivity Techniques in Nonlinear Control. Communication and Control Engineering. Springer Verlag.

van der Schaft, A. J. and B. M. Maschke (2002). Hamiltonian formulation of distributed parameter systems with boundary energy flow. Journal of Geometry and Physics 42(12), 166-194. 